\title{
$\alpha$-Adrenoceptor Subtype in the Hypothalamic Paraventricular Nucleus Involved in the Regulation of Urine Production: Comparison between Wistar Kyoto and Spontaneously Hypertensive Rats
}

\author{
Hiromi Tsushima and Seigo Fujimoto \\ Department of Pharmacology, Nagoya City University Medical School, Kawasumi, Mizuho-ku, Nagoya 467, Japan
}

Received December 2, 1996 Accepted March 3, 1997

\begin{abstract}
Microinjections of $\alpha$-adrenoceptor agonists into the paraventricular nucleus (PVN) of Wistar Kyoto rats (WKY) decreased the urine outflow rate in dose- and time-dependent manners. The order of the antidiuretic potency is norepinephrine (an $\alpha$-agonist) $>$ phenylephrine (an $\alpha_{1}$-agonist) $\gg$ clonidine (an $\alpha_{2^{-}}$ agonist). The phenylephrine-induced effect was inhibited by WB4101 (an $\alpha_{1}$-antagonist), but not by yohimbine (an $\alpha_{2}$-antagonist). $d\left(\mathrm{CH}_{2}\right)_{5}$-D-Tyr(Et)VAVP (a vasopressin antagonist) blocked the antidiuretic effect of phenylephrine. In spontaneously hypertensive rats (SHR), norepinephrine and phenylephrine produced weaker antidiuretic effects than in WKY. These findings suggest that the $\alpha_{1}$-subtype of the PVN decreases urine production mediated through vasopressin release. This mechanism is more feeble in SHR than WKY.
\end{abstract}

Keywords: $\alpha_{1}$-Adrenoceptor, Paraventricular nucleus, Vasopressin

Spontaneously hypertensive rats (SHR) have frequently been used as a model animal for essential hypertension in humans. It had been suggested that catecholaminergic neurons of the hypothalamic paraventricular nucleus (PVN) are involved in the development and/or maintenance of hypertension in SHR (1-5). In the PVN of SHR, there are increased synthesis and release of norepinephrine as compared to Wistar Kyoto rats (WKY) $(2,3,5)$. Since the PVN localizes the cell bodies of the vasopressin-containing neurons and synthesizes precursors of vasopressin, we have studied effects of $\hat{\beta}$ adrenoceptor agonists injected into the PVN of SHR on the urine outflow rate (6). We found that 1) the $\beta$ adrenoceptor whose stimulation decreased urine production in the PVN of SHR was the $\hat{\beta}_{2}$-subtype, although it was unclear in the control rats, 2) $\beta$-adrenoceptormediated decrease in urine outflow in SHR was similar to that in WKY and 3) this mechanism was not mediated through vasopressin release. In this study, we compared the effects of $\alpha$-adrenoceptor agonists injected into the PVN on urine outflow in SHR and WKY.

Male SHR (17- to 23-week-old) and age-matched WKY inbred in our laboratory (12-hr light/dark cycle, $23 \pm 1{ }^{\circ} \mathrm{C}$ ) were used. Their origin was the Okamoto-Aoki strain. The rats, starved for $17 \mathrm{hr}$, were orally administered tap water $(5 \mathrm{ml} / 100 \mathrm{~g}$ body weight) followed by, 45 min later, the same volume of $12 \%$ ethanol for anesthesia. After the trachea, urinary bladder and jugular vein of the animals were cannulated with polyethylene tubes, they were immobilized in a stereotaxic instrument. During the experiment, Locke's solution containing 3\% ethanol was infused at $0.1 \mathrm{ml} / \mathrm{min}$ through the jugular vein cannula to maintain anesthesia and urinary volume for several hours. Urinary drops flowing from the bladder cannula were continuously counted with a photoelectric drop counter. When the urine outflow rate became a constant level, a stainless steel cannula (o.d.: $200 \mu \mathrm{m}$ ) connected to a microsyringe was inserted into the PVN (coordinates: $5.6 \mathrm{~mm}$ anterior from the lambda, $0.3 \mathrm{~mm}$ to the right from the midline, $7.8 \mathrm{~mm}$ ventral from the dural surface) for the administrations of norepinephrine bitartrate (NE), phenylephrine hydrochloride (PHE), methoxamine hydrochloride (MET), clonidine hydrochloride (CLO), yohimbine hydrochloride (YOH) (Sigma Chemical Co., St. Louis, MO, USA) and 2-(2,6dimethoxyphenoxyethyl)aminomethyl-1,4-benzodioxane hydrochloride (WB4101; Research Biochemicals, Inc., Natick, MA, USA) (1 $\mu 1)$. 1-(Mercapto- $\beta, \beta$-cyclopentamethylene propionic acid)2-( $O$-ethyl)D-tyrosine, 4-valine, arginine-vasopressin $\left(d\left(\mathrm{CH}_{2}\right)_{5}\right.$-D-Tyr(Et)VAVP; a gift from Prof. K.G. Hofbauer, Cardiovascular Division, Ciba-Geigy, Ltd., Basel, Switzerland) (7) and Arg- 


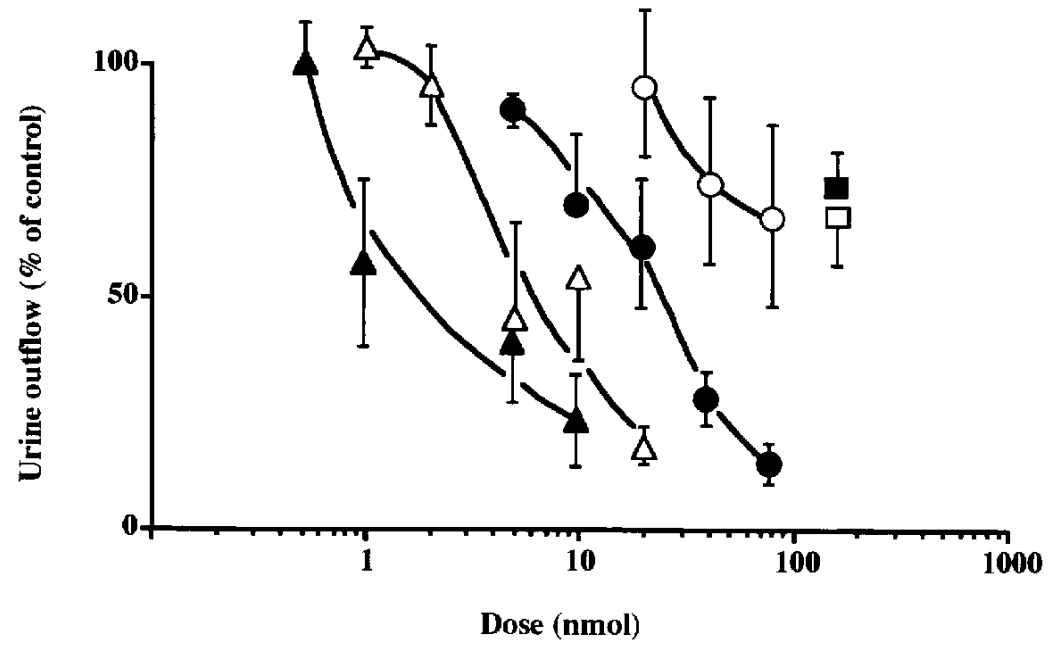

Fig. 1. Dose-effect curves for antidiuresis induced by microinjection of the $\alpha$-adrenoceptor agonists into the PVN of SHR and WKY. The abscissa shows the dose in nmol of phenylephrine (SHR: $\bigcirc$, WKY: $)$ ), norepinephrine (SHR: $\triangle$, WKY: $\Delta$ ) and clonidine (SHR: [, WKY: $\square$ ). The ordinate indicates the maximum decrease in the urine outflow rate, at 20 or 30 min after the administration, expressed as a percentage of the control level (urine outflow for $10 \mathrm{~min}$ before drug administration). The values are the mean \pm S.E.M. of 3-9 experiments.

vasopressin (Grade IV, Sigma) were intravenously injected through the jugular vein cannula $(0.1 \mathrm{ml})$. All drugs were dissolved in saline.

In WKY, NE (a non-selective $\alpha$-adrenoceptor agonist) and PHE (an $\alpha_{1}$-agonist), when injected into the PVN, produced dose-dependent antidiuresis (Fig. 1). The antidiuretic effect of $\mathrm{NE}$ at $10 \mathrm{nmol}$ began to appear within 5 min after the administration, the maximum effects were observed at 20-30 min after the administration, and the urine outflow rate recovered to the control level (the urine outflow rate before the drug administration) at $60 \mathrm{~min}$ after the administration. Microinjection of CLO (an $\alpha_{2^{-}}$ agonist) at $160 \mathrm{nmol}$ into the PVN was also antidiuretic. The order of their potencies was NE $>$ PHE $\gg$ CLO. In SHR, NE and PHE were less potent as antidiuretic agents. The antidiuretic effect of CLO in SHR was similar to that in WKY (Fig. 1). In WKY, WB4101 (an $\alpha_{1}$-antagonist), injected at $20 \mathrm{nmol}$ into the PVN, inhibited the PHE ( $40 \mathrm{nmol})$-induced antidiuresis. The small antidiuretic response still remained at $20 \mathrm{~min}$, and the diuretic response appeared at 40 and $50 \mathrm{~min}$ after the administration (Fig. 2A). Microinjection of WB4101 alone into the nucleus slightly decreased the urine outflow rate $\left(54 \pm 16.3 \% \%^{*}\right.$ at $30 \mathrm{~min}, 75 \pm 18.6 \%$ at $40 \mathrm{~min}, 86 \pm$ $17.6 \%$ of the control level at $50 \mathrm{~min}$ after the administration; ${ }^{*} \mathrm{P}<0.05$ vs the saline-injected group at the corresponding time-points). Intra-PVN injection of $\mathrm{YOH}$ (an $\alpha_{2}$-antagonist) at $10 \mathrm{nmol}$ did not inhibit the PHEinduced antidiuretic effect (Fig. 2A). When $d\left(\mathrm{CH}_{2}\right)_{5}$-D$\operatorname{Tyr}(\mathrm{Et})$ VAVP $(16.7 \mu \mathrm{g}$, a non-specific vasopressin receptor antagonist, Ref. 7) was injected intravenously, PHE did not cause antidiuresis, but caused marked diuresis having a 70-min duration (Fig. 2B). Another $\alpha_{1}$-agonist, MET at $40 \mathrm{nmol}$ elicited the antidiuretic effect with a time course similar to that of the PHE $(40 \mathrm{nmol})$-induced effect. The MET-induced antidiuresis was converted to diuresis after intravenous injection of $d\left(\mathrm{CH}_{2}\right)_{5}$-DTyr(Et)VAVP. The vasopressin receptor antagonist alone showed no effect on the urine outflow rate. We comfirmed that the vasopressin antagonist completely blocked antidiuresis induced by intravenous injection of $4 \mathrm{mU}$ vasopressin under the same experimental condition (8).

The present results suggested that the $\alpha$-adrenoceptor in the PVN for the decrease in urine production was the $\alpha_{1}$-subtype in ethanol-anesthetized hydrated rats. The weak antidiuresis of WB4101 was supposed to be nonspecific. The $\alpha_{1}$-adrenoceptor agonist-induced antidiuresis was mediated through vasopressin release since the PHE- and MET-induced antidiuretic effects were inhibited by the vasopressin receptor antagonist. Veltmar et al. suggested that the third $\alpha$-adrenoceptor subtype, neither $\alpha_{1}$ - nor $\alpha_{2}$-subtype, of the PVN decreases vasopressin release in unanesthetized rats (9). This shows a possibility that vasopressin release is regulated by various mechanisms and a certain condition activates the appropriate, suitable mechanism for regulation. That is, $\alpha_{1}$ - and the third $\alpha$-adrenoceptors seem to predominantly function under low and high/normal concentration of vasopressin in plasma, respectively. The $\alpha_{1}$-agonists were diuretic in the rats pretreated with $d\left(\mathrm{CH}_{2}\right)_{5}$-D-Tyr(Et)VAVP. Antidiuretic effects of oxotremorine (10), substance $P$ (11), angiotensin II (12) and the peptidase inhibitors (13) were 


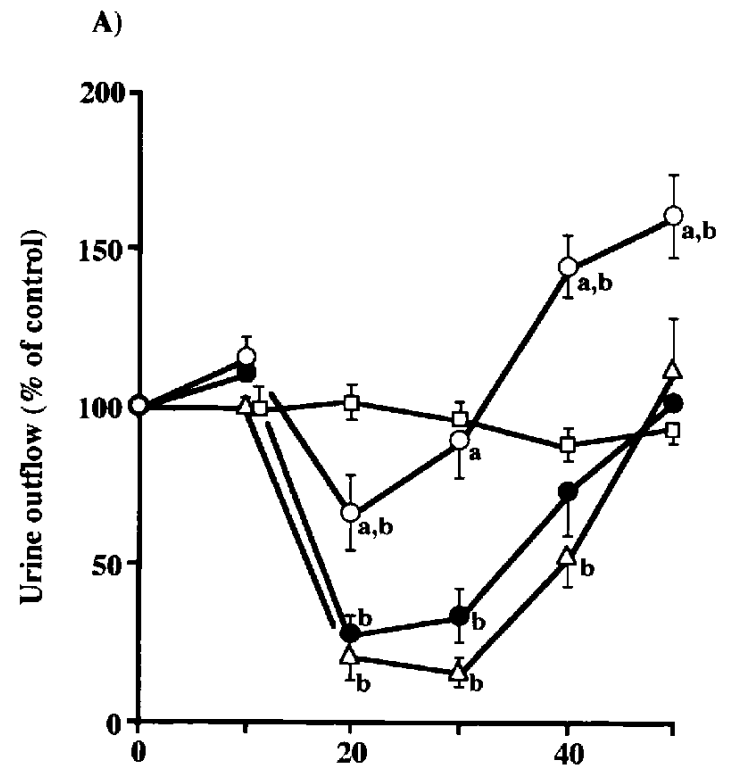

B)

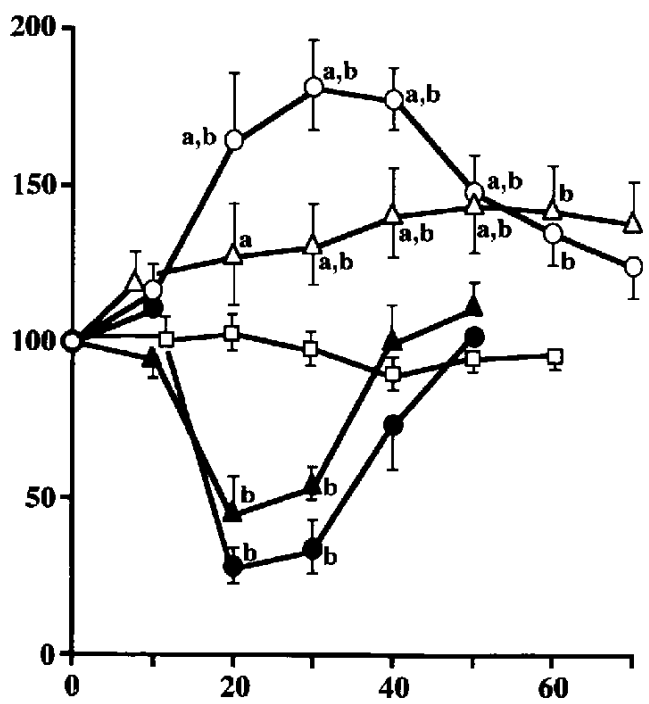

Time after microinjection (min)

Fig. 2. Inhibitory effects of the $\alpha$-adrenoceptor antagonists (A) and the vasopressin receptor antagonist (B) on the $\alpha_{1}$-adrenoceptor agonist-induced antidiuresis in WKY. The abscissa and ordinate show the time in min after administration of $\alpha_{1}$-adrenoceptor agonists and the change in the urine outflow rate expressed as a percentage of the control level, respectively. A) : $40 \mathrm{nmol}$ phenylephrine (PHE), $\bigcirc: 20 \mathrm{nmol}$ WB4101 + PHE, $\triangle: 10 \mathrm{nmol}$ yohimbine + PHE, $\square:$ saline; B) O: $40 \mathrm{nmol}$ PHE, A: $40 \mathrm{nmol}$ methoxamine (MET), $: 16.7 \mu \mathrm{g} d\left(\mathrm{CH}_{2}\right)_{5}$-D-Tyr(Et)VAVP + PHE, $\triangle: d\left(\mathrm{CH}_{2}\right)_{s}$-D-Tyr(Et)VAVP + MET, $\square$ : saline. Pretreatment with the antagonists was performed at $30-50 \mathrm{~min}$ before the agonist administration. The values are the mean \pm S.E.M. of $3-6$ experiments. ${ }^{a} \mathbf{P}<0.05$ vs the closed symbols, ${ }^{b} \mathbf{P}<0.05$ vs the saline-treated group $(\mid)$ at the corresponding time-points. Significant differences were determined by Scheffe's test following ANOVA.

completely blocked by the vasopressin receptor antagonist and no diuretic effect appeared. Also, PHE caused the diuretic effects following the weak antidiuretic effects after the pretreatment with WB4101. These phenomena do not seem simply a rebound effect. We speculated that the $\alpha_{1}$-agonists have two opposite effects, antidiuretic and diuretic, and that diuretic effects are exposed under certain conditions which only inhibit the antidiuretic effects. The antidiuretic effects were mediated through increased vasopressin release via $\alpha_{1}$-adrenoceptors as described above; however, mechanisms underlying the diuresis are yet undefined. At least, the diuretic effects did not involve activation of $\alpha_{1}$-adrenoceptors. The following can be proposed as possible mechanisms: 1) indirect effects to neurons in the hypothalamus that modulate function of the kidney (14), 2) effects resulting from the interaction between the anesthetic, ethanol, and the $\alpha_{1}$-agonists and 3) non-specific effects of the $\alpha_{1}$-agonists. The regulation via the $\alpha_{1}$-subtype in SHR is weaker than that in WKY. The reason is probably down-regulation of $\alpha_{1}$-receptors in the PVN of SHR. It was demonstrated that activities of catecholaminergic neurons (1) and tyrosine hydroxylase (3) and catecholamine release $(2,5)$ increase in the PVN of SHR more than WKY. Therefore, $\alpha_{1}$-receptors in the
PVN of SHR may be down-regulated.

In conclusion, 1) stimulation of the $\alpha_{1}$-adrenoceptor subtype in the hypothalamic PVN decreases the urine production via vasopressin release and 2) this mechanism is more feeble in SHR than WKY.

\section{REFERENCES}

1 Kubo $\mathrm{T}$ and Hashimoto M: Effects of intraventricular and intraspinal 6-hydroxydopamine on blood pressure of spontaneously hypertensive rats. Arch Int Pharmacodyn Ther 232, 166- 176 (1978)

2 Qualy JM and Westfall TC: Release of norepinephrine from the paraventricular hypothalamic nucleus of hypertensive rats. Am J Physiol 254, H993-H1003 (1988)

3 Morris M and Bennet BA: An analysis of catecholaminergic and peptidergic cells in paraventricular and arcuate cultures in the hypertensive rat. Eur J Pharmacol 183, 842 (1990)

4 Takeda K, Nakata T, Takesako T, Itoh H, Hirata M, Kawasaki S, Hayashi J, Oguro M, Sasaki S and Nakagawa M: Sympathetic inhibition and attenuation of spontaneous hypertension by PVN lesions in rats. Brain Res 543, 296-300 (1991)

5 Woo ND, Mukherjee K and Ganguly PK: Norepinephrine levels in paraventricular nucleus of spontaneously hypertensive rats: role of neuropeptide Y. Am J Physiol 265, H893-H898 (1993)

6 Tsushima $H$, Fujimoto $S$ and Matsuda $T$ : Effects of $\beta_{1^{-}}$and 
$\hat{\beta}_{2}$-adrenoceptor agonists applied into the hypothalamic paraventricular nuclei of spontaneously hypertensive rats on urine production. Jpn J Pharmacol 64, 201-207 (1994)

7 Hofbauer KG, Mah SC and Opperman JR: Chronic blockade of vasopressin receptors in rats. J Cardiovasc Pharmacol 8 , Supp 7, S56-S60 (1986)

8 Tsushima $H$, Mori $M$ and Matsuda T: Effects of D-Ala ${ }^{2}-D-$ Leu ${ }^{5}$-enkephalin, microinjected into the supraoptic and paraventricular nuclei, on urine outflow rate. Jpn J Pharmacol $63,181-186(1993)$

9 Veltmar A, Culman J, Qadri F, Rascher W and Unger T: Involvement of adrenergic and angiotensinergic receptors in the paraventricular nucleus in the angiotensin II-induced vasopressin release. J Pharmacol Exp Ther 263, 1253-1260 (1992)

10 Mori $M$, Tsushima $\mathrm{H}$ and Matsuda $\mathrm{T}$ : Antidiuretic effects of oxotremorine microinjected into the hypothalamic supraoptic and paraventricular nuclei in a water-loaded and ethanol- anesthetized rat. Jpn J Pharmacol 35, 27-36 (1984)

11 Mori M, Tsushima $\mathrm{H}$ and Matsuda T: Substance $\mathrm{P}$ injected into the hypothalamic supraoptic nucleus causes antidiuresis through the release of arginine-vasopressin in water-loaded and ethanol-anesthetized rats. Jpn J Pharmacol 62, 49 - 56 (1993)

12 Tsushima $\mathrm{H}$, Mori $\mathrm{M}$ and Matsuda $\mathrm{T}$ : Microinjections of angiotensin II into the supraoptic and paraventricular nuclei produce potent antidiureses by vasopressin release mediated through adrenergic and angiotensin receptors. Jpn J Pharmacol 66, 241-246 (1994)

13 Tsushima H, Mori $M$ and Matsuda T: Peptidase inhibitorinduced antidiuresis mediated through angiotensin and opioid receptors in the rat hypothalamus. Jpn J Pharmacol 71, 61-71 (1996)

14 Ciriello $\mathbf{J}$ and Calaresu FR: Hypothalamic projections of renal afferent nerves in the cat. Can J Physiol 58, 574-576 (1980) 